Issued by Sandia National Laboratories, operated for the United States Department of Energy by Sandia Corporation.

NOTICE: This report was prepared as an account of work sponsored by an agency of the United States Government. Neither the United States Government, nor any agency thereof, nor any of their employees, nor any of their contractors, subcontractors, or their employees, make any warranty, express or implied, or assume any legal liability or responsibility for the accuracy, completeness, or usefulness of any information, apparatus, product, or process disclosed, or represent that its use would not infringe privately owned rights. Reference herein to any specific commercial product, process, or service by trade name, trademark, manufacturer, or otherwise, does not necessarily constitute or imply its endorsement, recommendation, or favoring by the United States Government, any agency thereof, or any of their contractors or subcontractors. The views and opinions expressed herein do not necessarily state or reflect those of the United States Government, any agency thereof, or any of their contractors.

Printed in the United States of America. This report has been reproduced directly from the best available copy.

Available to DOE and DOE contractors from

U.S. Department of Energy

Office of Scientific and Technical Information

P.O. Box 62

Oak Ridge, TN 37831

Telephone: (865)576-8401

Facsimile: (865)576-5728

E-Mail: reports@adonis.osti.gov

Online ordering: http://www.doe.gov/bridge

Available to the public from

U.S. Department of Commerce

National Technical Information Service

5285 Port Royal Rd

Springfield, VA 22161

Telephone: (800)553-6847

Facsimile: (703)605-6900

E-Mail: orders@ntis.fedworld.gov

Online order: http://www.ntis.gov/ordering.htm

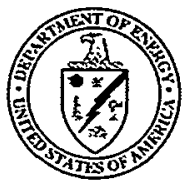




\section{DISCLAIMER}

Portions of this document may be illegible in electronic image products. Images are produced from the best available original document. 
SAND2000-3070

Unlimited Release

Printed December 2000

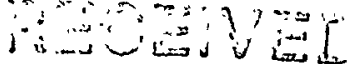

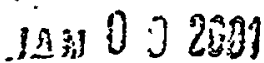

(2)

\title{
Application of Roll-isolated Inertial Measurement Units to the Instrumentation of Spinning Vehicles
}

\author{
Mark E. Beader, Editor \\ Guidance Subsystems Department \\ Sandia National Laboratories \\ P.O Box 5800 \\ Albuquerque, NM 87185-0501
}

\begin{abstract}
Roll-isolated inertial measurement units are developed at Sandia for use in the instrumentation, guidance, and control of rapidly spinning vehicles. Roll-isolation is accomplished by supporting the inertial instrument cluster (gyros and accelerometers) on a single gimbal, the axis of which is parallel to the vehicle's spin axis. A rotary motor on the gimbal is driven by a servo loop to null the roll gyro output, thus inertially stabilizing the gimbal and instrument cluster while the vehicle spins around it. Roll-isolation prevents saturation of the roll gyro by the high vehicle spin rate, and vastly reduces measurement errors arising from gyro scale factor and alignment uncertainties. Nine versions of Sandia-developed rollisolated inertial measurement units have been flown on a total of 27 flight tests since 1972.
\end{abstract}




\section{ACKNOWLEDGEMENTS}

Contributors to this report at Sandia National Laboratories include: Mark Beader, Joe Lucero, Jeff Wilcoxen, Mike Heck, and Stew Kohler of the Guidance Subsystems Department; Earl Creel of the Navigation, Guidance, and Control Department; Bev Sturgis of the Aerosciences and Computational Fluid Mechanics Department; and Ed Talbot of the Enduring RV Systems Department. 


\section{TABLE OF CONTENTS}

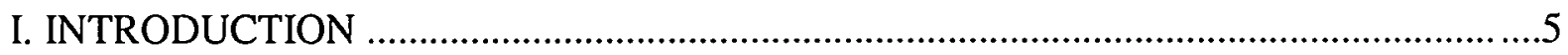

II. HISTORY OF ROLL-ISOLATED IMUS AT SANDIA .............................................

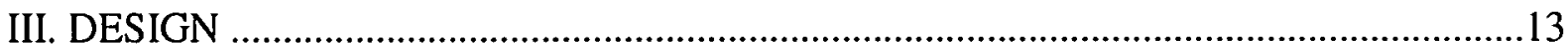

IV. ADVANTAGES AND DISADVANTAGES OF ROLL-ISOLATION .........................17

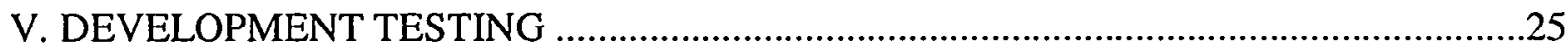

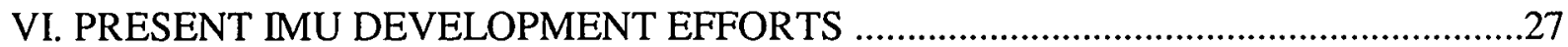

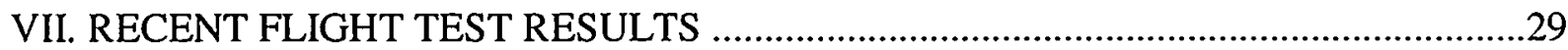

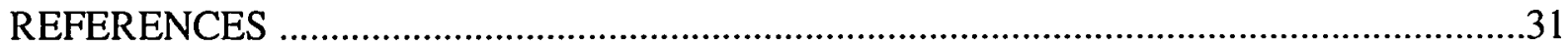

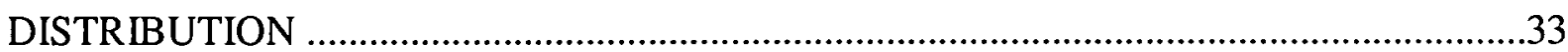


This page intentionally left blank 


\section{INTRODUCTION}

Roll-isolated inertial measurement units (IMUs) are developed at Sandia for use in the instrumentation, guidance, and control of rapidly spinning vehicles. Examples of such vehicles include spin-stabilized reentry vehicles and sounding rockets (see below).
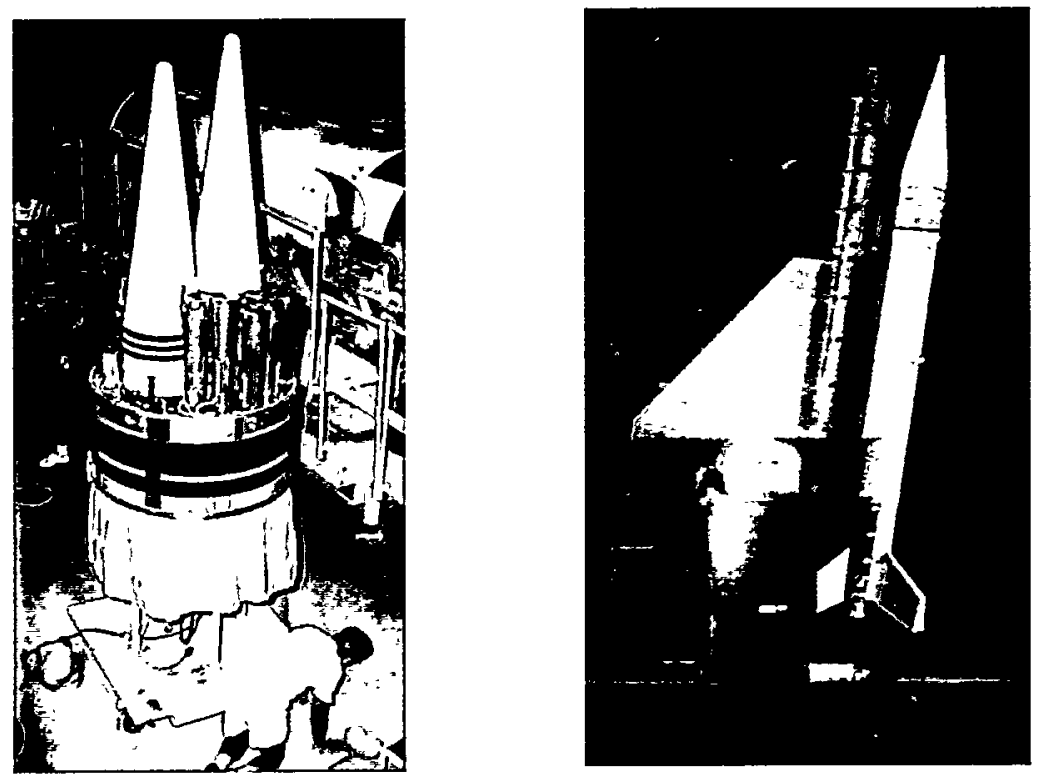

Roll-isolation is accomplished by supporting the inertial instrument cluster (gyros and accelerometers) on a single gimbal, the axis of which is parallel to the vehicle's spin axis. A rotary motor on the gimbal is driven by a servo loop to null the roll gyro output, thus inertially stabilizing the gimbal and instrument cluster while the vehicle spins around it. This mechanization is commonly referred to in literature as a "single-gimbal platform". The motor is required to cancel the gimbal bearing torque which would otherwise drag the gimbal in the direction of vehicle roll. Roll-isolation prevents saturation of the roll gyro by the high vehicle spin rate, and greatly reduces measurement errors arising from gyro scale factor and alignment uncertainties.

An angle transducer is included as part of the gimbal mechanization to measure the roll angle between the instrument cluster and the vehicle. This measurement, along with the outputs of the gyros and accelerometers, allows a complete determination of the vehicle motion. The attitude calculations of the instrument cluster are identical to those for a strapdown (i.e.,strapped-down, not isolated) IMU in which the output of the roll gyro is very small. Vehicle attitude differs from that of the instrument cluster only in roll. This difference is determined by reading the output of the roll position transducer on the gimbal.

Nine different versions of roll-isolated inertial measurement units have been developed at Sandia since 1972. Relative to its predecessors, each new version in the series incorporates improvements, mainly in inertial sensor technology, to accomplish improvements in performance, size, and/or cost. Sandia-developed roll-isolated IMUs have been flight tested on a total of 27 occasions since 1972. 
This page intentionally left blank 


\section{HISTORY OF ROLL-ISOLATED IMUS AT SANDIA}

The use of a roll gimbal to isolate gyroscopes from the high roll rates encountered in spinstabilized rocket payloads dates back to the early 1960s. During the atmospheric test readiness program, Sandia's field test organization, in collaboration with Whitaker Gyro, developed the MARS (Miniature Attitude $\underline{\text { Reference }}$ System) platform to provide an all-attitude angular reference to orient sensors (optical, infrared, radiation) aboard spinning instrument payloads. The MARS used a pair of two-axis free gyroscopes mounted on a motor-driven roll-axis gimbal to achieve all-attitude capability. Hundreds of these systems have been flown over the past four decades in Sandia and NASA experiments. An upgraded version of the MARS system is still in production and available from Space Vector Corporation.

In the late 1960s, Sandia research initiatives in maneuvering reentry vehicles (MaRVs) led to the development of a high-G roll reference. A two-axis (roll and pitch) stable platform was designed using high-G, rate-integrating gyros from Honeywell (Figure 1, below). This mechanization provided the capability to maintain a trajectory plane reference ("which way is up?") through launch and separation of maneuvering reentry vehicles deployed from spin-stabilized rockets. Experiments were conducted at the Tonopah Test Range, Nevada (1972) and at the Kauai Test Facility, Hawaii (1974) using the two-axis platform as a reference for "open-loop" maneuvers from the ballistic trajectory.

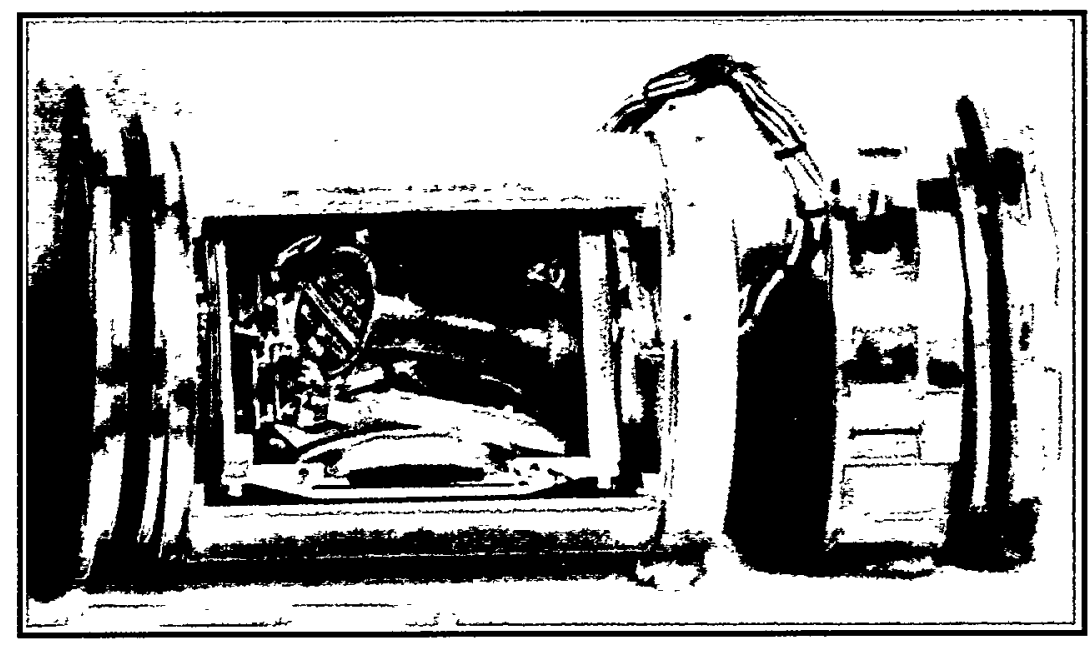

Figure 1: Two-axis Attitude Reference, circa 1972.

In the mid-1970s, interest developed in providing more accurate guidance of MaRVs. The availability of onboard strapped-down navigation computers led to the development of rollstabilized IMUs with full (3-axis) complements of gyros and accelerometers and inertial navigation capability for "closed loop" control of MaRV position during reentry. Between 1976 and 2000, nine different roll-isolated IMUs were developed by Sandia. In some cases, these developments were joint efforts with industry participants. A chronological listing and photos of these IMUs follow. 


\section{Chronology of Roll-isolated IMUs Developed at Sandia}

1. 1976 - RIMS I (Roll-isolated Inertial Measurement System I): Honeywell GG1009E rateintegrating gyros, Sandia high-g accelerometers. (References 1\&2).

2. 1978 - RIMS II: -- Incosym Mod IIIE dynamically tuned gyros (DTGs), Sundstrand QA 1200 accelerometers. Joint development with Space Vector Corp.

3. 1981 - Wellbore Inertial Navigation System: Incosym Mod IIIE dynamically tuned gyros (DTGs), Sundstrand QA 1200 accelerometers. (Reference 3).

4. 1984 - Mini-RIMS A/RIMDAC: Incosym Mod XVII dynamically tuned gyros (DTGs), Sundstrand Model 2180 (Mini-Pal) accelerometers. Joint development with Incosym Inc. (Reference 4).

5. 1987 - Mini-RIMS C: Incosym Mod XVII dynamically tuned gyros (DTGs), Sundstrand QA 1200 accelerometers. Joint development with Incosym Inc.

6. 1989 - RLGA (Ring Laser Gyro Assembly): Honeywell GG1320 RLGs, Sundstrand QA1200 accelerometers. Joint development with Honeywell Inc. (Reference 5)

7. 1991 - DMARS (Digital Miniature Attitude Reference System): Condor Pacific T-100 dynamically tuned gyros (DTGs), Sundstrand QA-700 Accelerometers. Joint development with Inertial Science Inc. (Reference 6)

8. 1998 - GLN-200 (Gimbaled Litton LN-200 IMU): Litton fiber optic gyros (FOGs), Litton silicon accelerometers (SiAc's $\left.{ }^{\mathrm{TM}}\right)$. (References $7 \& 8$ ).

9. 2000 - QLN-2000 (GLN-200 with QA 2000 Accelerometer Triad): Litton fiber optic gyros (FOGs), Litton silicon accelerometers (SiAc's ${ }^{\text {TM }}$ ), Sundstrand QA-2000 accelerometers. (Reference 7).

10. 2001 (Under development) - QLN-MAC (QLN-2000 with Sandia Miniature Airborne Computer) 


\section{Photo History of Sandia Roll-isolated IMUs}

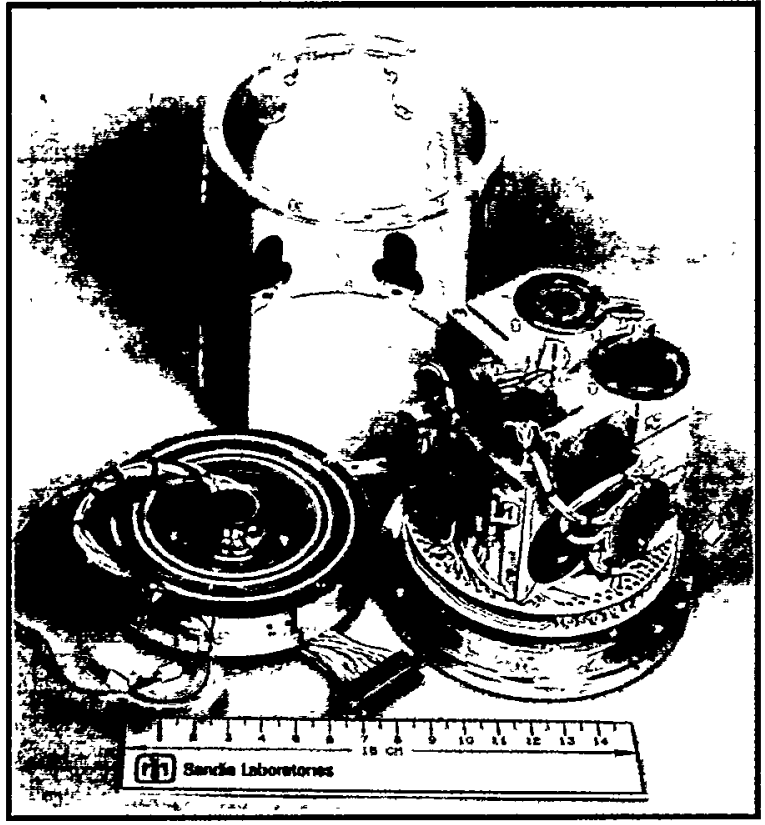

RIMS I (1976)

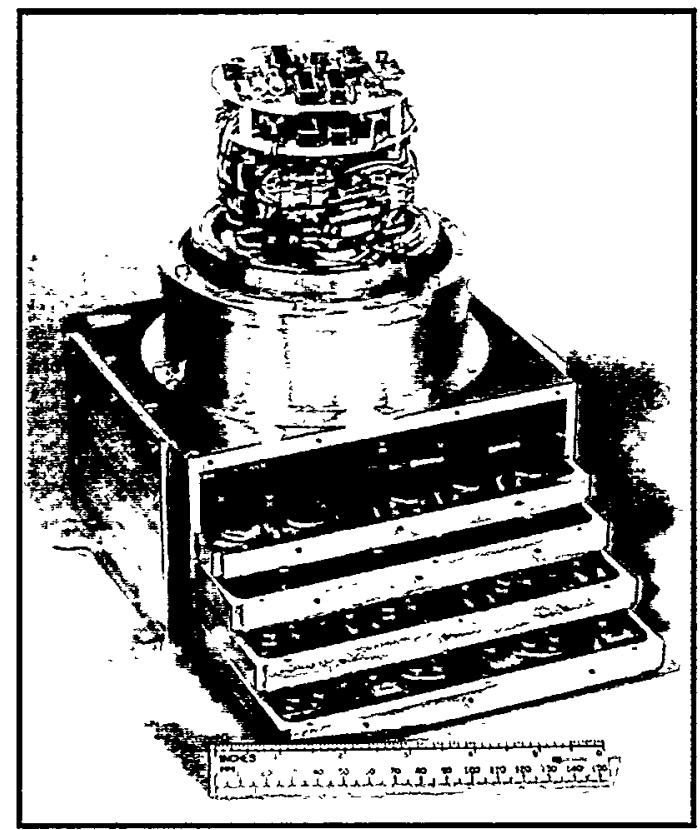

RIMS II (1978)

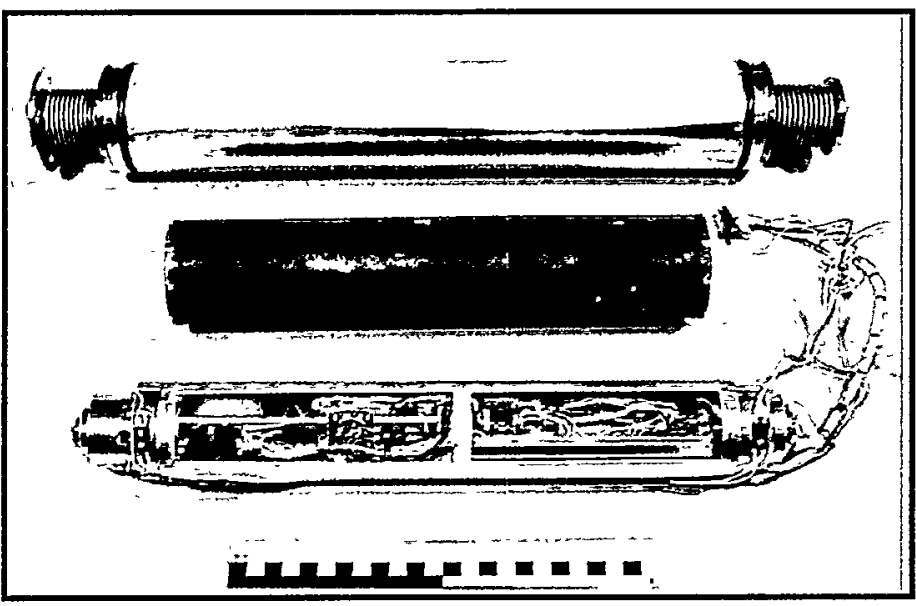

Wellbore Inertial Navigation System (1981)

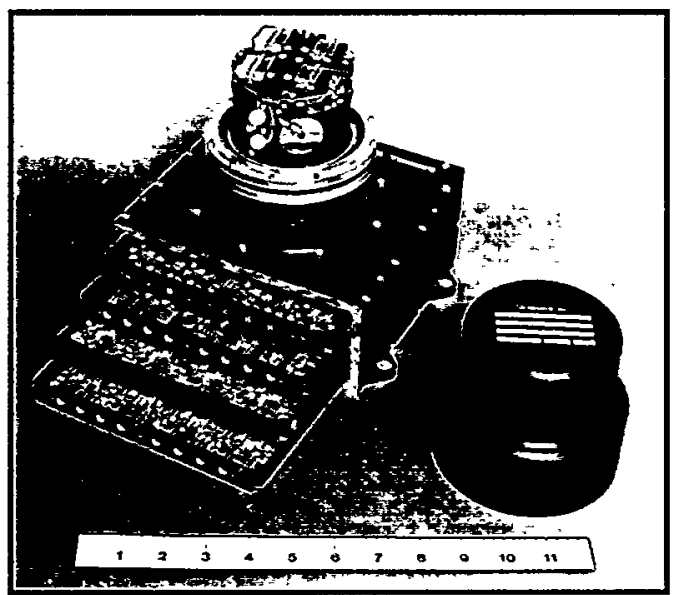

Mini-RIMS A (1984) 


\section{Photo History of Sandia Roll-isolated IMUs}

(continued)

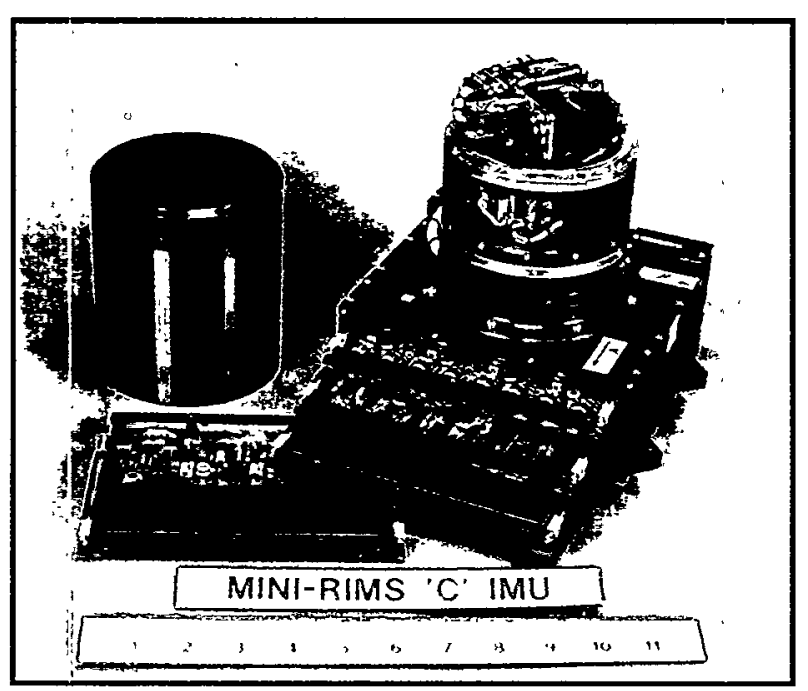

Mini-RIMS C (1987)

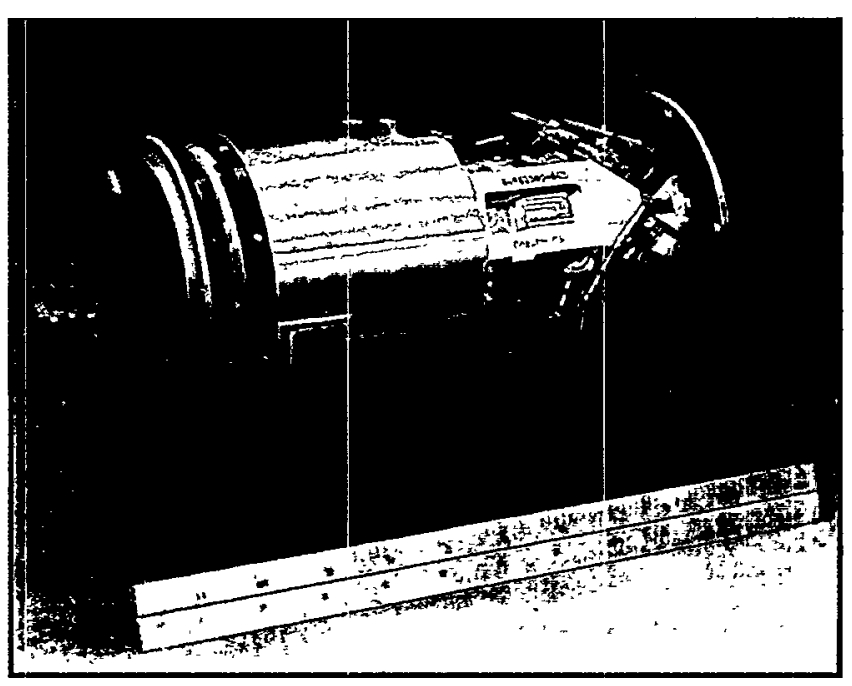

RLGA-RIMS (1989)

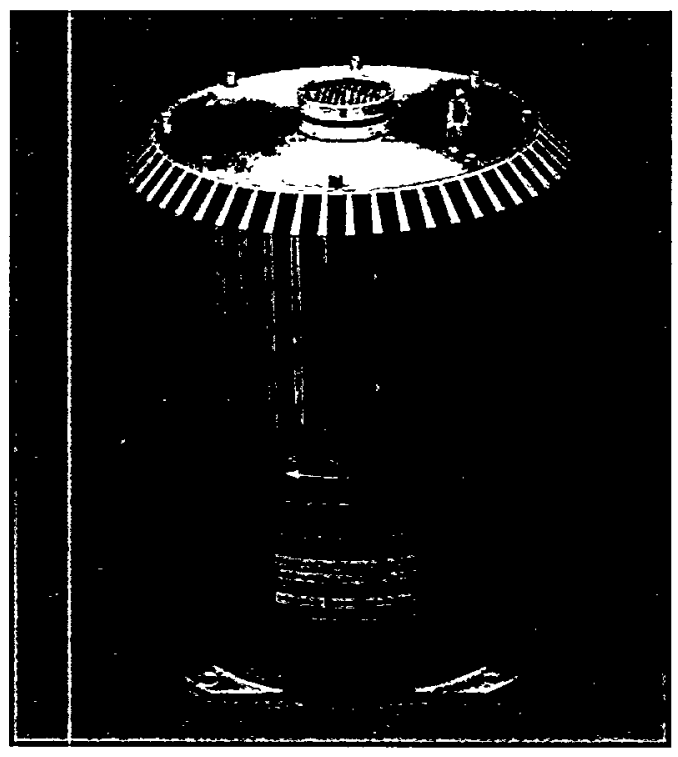

DMARS (1992)

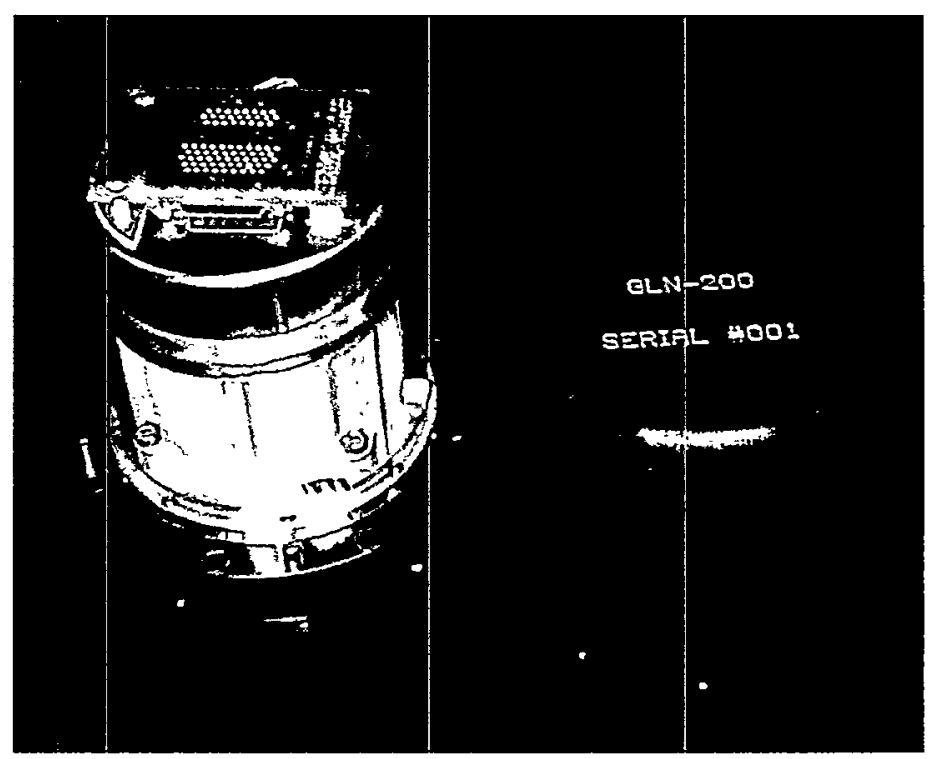

GLN-200 (1998) 


\section{Flight Test History}

The table below gives flight tests and associated IMUs for the 27 occasions in which Rollisolated IMUs were used. Vehicles include reentry vehicles (RVs), maneuvering reentry vehicles (MaRVs), sounding rockets (SRs), and various exoatmospheric test vehicles flown in support of the Strategic Defense Initiative (SDI) and missile defense (MD)-related experiments. The SDI and MD test vehicles were typically spin-stabilized, and were deployed exoatmospherically by post-boost vehicles (PBVs).

\section{Flight Test History - Sandia Roll-isolated IMUs}

Note: VAFB: Vandenberg AFB (CA), KTF: Kauai test Facility (HI), KMR: Kwajalein Missile Range (South Pacific), WSMR: White Sands Missile Range (NM)

\begin{tabular}{|l|l|l|l|}
\hline Year & Vehicle & Test site & IMU \\
\hline 1976 & RV (SAMAST 03) & VAFB to KMR & RIMS I \\
\hline 1979 & MRV (SWERVE I) & KTF & RIMS I \\
\hline 1980 & MRV (SWERVE II) & KTF & RIMS I \\
\hline 1983 & SDI (HOE I,II,III) & VAFB to KMR & RIMS II (3) \\
\hline 1984 & SDI (HOE IV, STV IV) & VAFB to KMR & RIMS II (3) \\
\hline 1985 & MRV (SWERVE III) & KTF & RIMS II \\
\hline 1987 & SR (STRYPI XI) & KTF & Mini-RIMS C \\
\hline 1989 & SR (Thorny Merit) & KTF & Mini-RIMS C (2) \\
\hline 1991 & SR (LACE) & KTF & MINI-RIMS C \\
\hline 1991 & SDI (ERIS) & VAFB to KMR & MINI-RIMS C (2) \\
\hline 1992 & SDI (ERIS) & VAFB to KMR & Mini-RIMS C \\
\hline 1993 & RV (RBIP) & Sub Launch (near FL to & RLGA-RIMS \\
& & Ascension Island) & \\
\hline 1995 & SR (CHOP) & WSMR & DMARS \\
\hline 1995 & SR (ETCE I, II) & KTF & DMARS (2) \\
\hline 1998 & MD (GBI, IFT-I, IA) & VAFB to KMR & Mini-RIMS A/RIMDAC (2) \\
\hline 1999 & MD (GBI, IFT-II) & VAFB to KMR & Mini-RIMS A/RIMDAC \\
\hline 1999 & MD (GBI, IFT-III) & VAFB to KMR & GLN-200 \\
\hline 2000 & MD (GBI, IFT-IV, V) & VAFB to KMR & GLN-200 \\
\hline 2000 & RV (W87 JTA, IDF-I) & VAFB to KMR & QLN-2000 \\
\hline
\end{tabular}


This page intentionally left blank 


\section{DESIGN}

Earlier versions of roll-isolated IMUs developed at Sandia have used either spinning wheel or ring laser gyros. The gyros and accelerometers were commercially available instruments and custom instrument cluster designs were required to provide the proper cylindrical form factor and to minimize size and weight. The most recent designs, the GLN-200 and QLN-2000, incorporate the Litton LN-200 IMU without modification. The availability of this IMU, already cylindrical, and very small and light ( 35 cubic inches and 1.5 pounds), enabled, for the first time, the use of a commercially available sensor assembly.

Common, key elements of roll-isolated IMU designs include gimbal bearings, vibration isolators, torque-motors, roll-position transducers, and the gimbal control circuitry. Design considerations and variations of these elements between IMUs are described briefly below.

Bearings - All Sandia roll-isolated IMU designs have employed gimbal bearings in angular contact, pre-loaded duplex pairs. This bearing geometry, available from several companies, provides an extremely stiff mechanical structure when the outer races of the bearing pair are pressed together. Resonant frequencies above $400 \mathrm{HZ}$ have been achieved for most Sandia rollisolated IMU designs. Maintaining a high resonant frequency of the roll gimbal structure is important, particularly for mechanical inertial instruments, to avoid excitation of gyro structural resonances, and to minimize high vibrational inputs to the gyro servo loops, which typically have a bandwidth of less than $100 \mathrm{~Hz}$. Depending on the weight and geometry of the bearingsupported IMU structure, both cantilevered designs (single duplex pair at on one end of the IMU) and "two-ended" (duplex pairs at each end of the IMU structure) configurations have been used. Examples of cantilevered design include RIMS II, Mini-RIMSA, and the GLN-200. "Twoended" design examples are RIMS I, the Wellbore Inertial Navigation System, Mini-RIMS C, DMARS, RLGA-RIMS, and the QLN-2000.

Vibration Isolators - All Sandia IMU designs have included some form of vibration isolation. This is required to minimize inertial instrument damage in mechanical shock, and to attenuate external vibrational inputs at the instrument and gimbal bearing resonant frequencies. Both ring and grommet type isolators have been used to provide rapid roll-off of external vibrations above $100 \mathrm{~Hz}$.

Torque Motors, Gimbal Position Transducers - All Sandia roll-isolated IMU designs have incorporated pancake-style, direct-drive, DC torque motors. With the exception of RIMS I, which used a potentiometer, pancake-style resolvers have been used exclusively to measure roll position.

Electronics - A block diagram of the electronics for a modern roll-isolated IMU is shown in Figure 2 below. This digital architecture has been used since high-throughput digital processors became available to perform gimbal control and input/output (I/O) functions. It was first implemented in the late 1980s in the roll-isolated RLGA-RIMS. Earlier systems employed analog circuitry for gimbal control. 


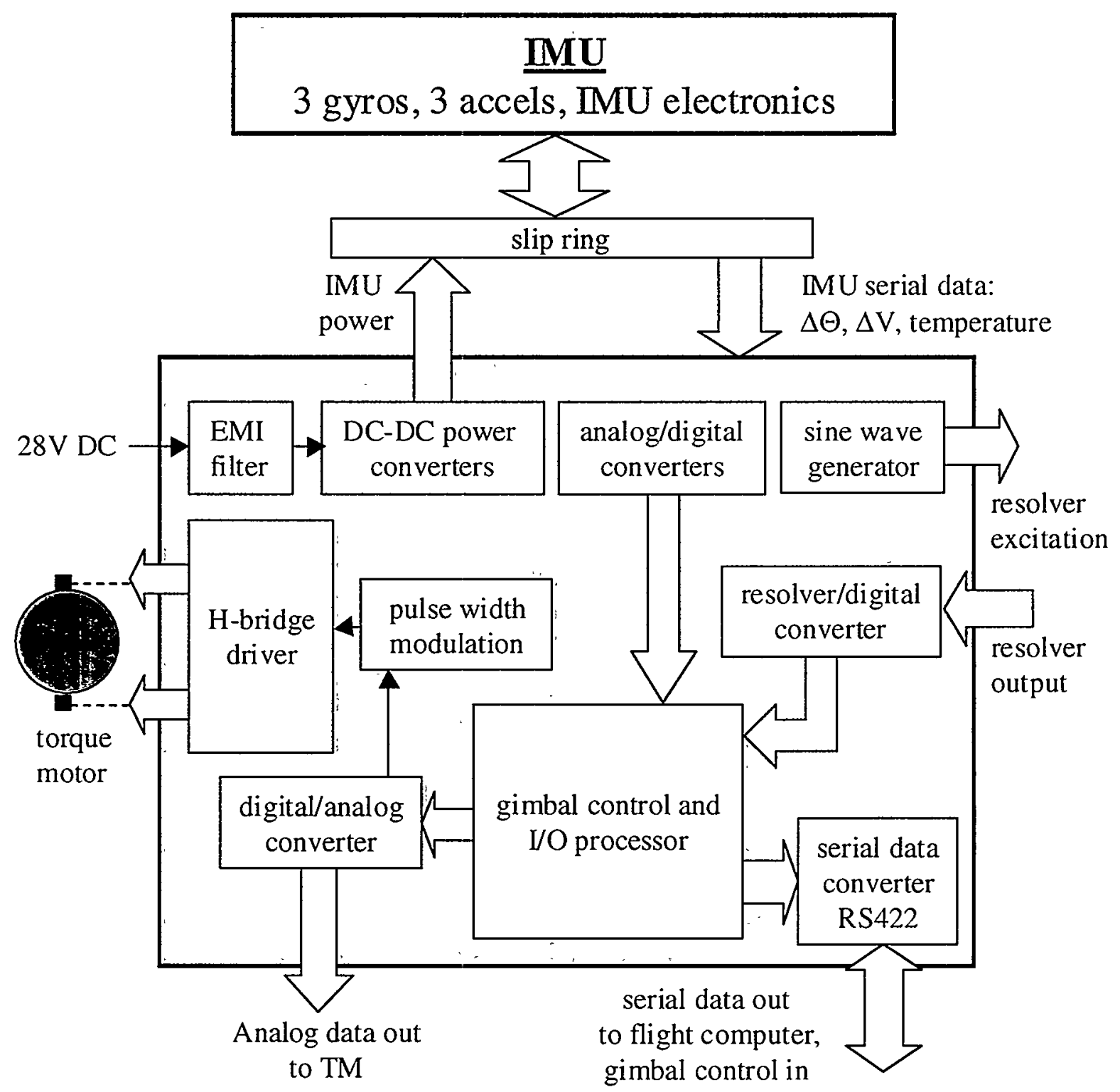

Figure 2: Block Diagram, Roll-Isolated IMU Electronics

The functions performed in the roll-isolated IMU circuitry are described below:

Power Conversion - Sandia roll-isolated IMUs operate from a 28 VDC battery. Commercially available power converter modules have been used in recent designs to provide the unique voltages required by the IMU, control processor, A/D, R/D, \& D/A (analog-to-digital, resolver-to digital, and digital-to-analog) converters, the pulse width modulator, and the sine wave generator for resolver excitation.

Input/Output (I/O) - Analog and digital data are provided on the roll-isolated IMU output connectors for monitoring IMU performance, and system diagnostics including supply voltage 
monitors, temperature monitors, and torque-motor drive. Digital serial data outputs include compensated gyro and accelerometer data from IMU data stream, and digitized resolver position and angular rate from the R/D converter. These outputs are available for attitude calculation and navigation by a flight computer, and for monitoring and/or autopilot control of the parent vehicle during flight. D/A converters are used to provide digitized outputs to telemetry (TM) of the analog gyro rates, accelerometers, and the torque motor drive. Gimbal control modes for caging, slewing, and inertial stabilization can also be input from a desktop or pre-launch control computer or from the onboard flight computer via an RS $422 \mathrm{~V} / O$ connector.

Gimbal stabilization and caging control - The major portion of the circuitry, and the control processor software, are associated with these functions. The high throughput capability of modern control and $\mathrm{V} / \mathrm{O}$ processors allows implementation of the gimbal caging loop at $800 \mathrm{~Hz}$, and gimbal stabilization at $400 \mathrm{~Hz}$ (typical). The processor serial $/ / O$ capability supports multiple channels of digitized analog TM data at $400 \mathrm{~Hz}$ to provide the data stream of gyro, accelerometer and resolver data to the flight computer. In addition, the I/O is also utilized for reading the 16bit R/D converter, and for providing an input to the D/A converter, which drives the pulse width modulators (PWMs) for torque motor drive.

Additional circuitry required to implement the gimbal control function includes a sine wave generator for resolver excitation, a 16- bit R/D converter which also provides an analog roll rate output, and the PWMs to drive the H-bridge torque motor driver.

Gimbal Control Modes - Gimbal control algorithms implemented in the processor include inertial stabilization, which nulls the roll gyro output from the IMU data stream by driving the PWM/H-Bridge/torque motor using the D/A converter. The control loop typically contains an integrator function to minimize inertial rate drift of the roll-stabilized IMU due to steady state roll rate inputs.

The second control mode is caging, which is achieved by driving the PWM/H-Bridge/Torquemotor from the difference between the commanded (desired) cage angle and the existing cage angle read from the R/D converter. The analog roll rate signal from the R/D is used for rate feedback in this control mode. The gimbal can be positioned to a precision of better than 0.01 degrees in the caging mode.

Slewing of the gimbal is a third control mode, which allows gimbal rotation at a various rates in either clockwise or counter-clockwise directions. This mode is useful for pre-launch testing of the system to verify proper operation of the IMU and the gimbal control circuitry, and for calibration of roll gyro scale factor and lateral gyro misalignments.

A more detailed mechanical and electrical description of a recent roll-isolated IMU design, the GLN-200, is provided in Reference 7. 
This page intentionally left blank 


\section{ADVANTAGES AND DISADVANTAGES OF ROLL-ISOLATION}

\section{Advantages}

Prevention of Roll Gyro Saturation - Sandia applications for inertial angular rate sensing include the instrumentation of rapidly spinning vehicles. Spin rates may exceed $20 \mathrm{rps}$, well above the rate capability of most gyros. In cases where the angular rate range is achievable, as in specially scaled versions of Litton's LN-200 IMU, the extended range is obtained at the cost of unacceptably low resolution (tenths of a degree/sec). Also, roll angle measurement errors due to other causes are unacceptable, as explained below.

Reduction of Roll Angle Measurement Errors - Gyro scale factor uncertainty may result in large measurement errors in strapdown IMUs that are subjected to large roll rates for extended periods of time. For example, a 2 rps roll rate may be expected to produce an error of 86 degrees after twenty minutes in the angle calculated from the output of a strapdown LN-200 IMU with an advertised scale factor uncertainty of $100 \mathrm{ppm}$. Even over short periods of time, a $20+$ rps spin rate would result in an error growth rate approaching a degree/sec. The corresponding error associated with a roll-isolated IMU is miniscule, because the gyro is not allowed to rotate, except that fractional-degree rotation may occur due to finite gimbal servo stiffness. Some vehicle roll angle measurement error occurs due to the gimbal angle resolver error, but this error, typically less than 0.05 degree, does not factor into navigation calculations (trajectory estimates) and does not increase with time.

Reduction of Errors Due to Gyro Misalignment - Lateral axis, or pitch and yaw, gyro input axis alignment uncertainty causes these gyros, in a strapdown IMU, to measure a component of roll rate. Strapdown IMUs utilizing ring laser gyros (RLGs) have been developed for spinning vehicle applications. Relative to other types of gyros, RLGs offer superior scale factor and input axis alignment stabilities of, typically, a few ppm and a few microradians respectively. In order to hold errors in spinning vehicle applications to an acceptable level, attempts have been made to calibrate scale factor and input axis alignment with an accuracy of parts-per-billion! This approach rapidly escalates into a modeling nightmare to characterize RLG scale-factor and alignment sensitivity to temperature, temperature gradients, magnetic fields, and elapsed time since calibration. Our experience at Sandia indicates that scale factor modeling to parts per billion is not practical for strapdown systems. The relative ease of roll-isolating an IMU has made this the approach of choice at Sandia for spinning vehicle applications.

Calibration and Self-Test Advantages - Standard roll gimbal control modes include "slewing" - the ability to rotate the gimbal at a desired rate both clockwise and counterclockwise, and "caging" where the gimbal can be positioned to 0.01 degree accuracy at any desired gimbal position. These control modes can prove extremely useful for pre-launch self-tests of most IMU functions and rapid calibration of many instrument error terms. This capability is particularly valuable when the IMU is mounted in a payload on the launch pad. For example, the caging feature can be used to rapidly derive horizontal (X, Y-axes) gyro biases by pointing these axes east and west to significantly reduce the time for azimuth alignment. Slewing the gimbal through many revolutions and re-caging allows rapid determination of the roll gyro scale factor and the 
pitch and yaw gyros' misalignment angles. The centrifugal force produced on the lateral accelerometers and angular rates on the gyros during slewing can also be used to verify their integrity at much higher rates and accelerations than those produced by gravity and earth rate. These self-test and calibration routines can be stored in the onboard flight computer and programmed to execute automatically when the inertial system is powered up prior to launch.

\section{Disadvantages}

Volume, Weight Increase - The increase in IMU size and weight due to the inclusion of a rollisolation capability can range from minor to significant. Two examples that illustrate these extremes are discussed below.

RLGA-RIMS: As shown in Figure 3 below, incorporating roll-isolation in the RLGA IMU added only one inch in length, 1.7 pounds, and 25 cubic inches of volume to the 11.6pound, 240-cubic inch, strapdown version. This represented a modest weight penalty of $15 \%$, and a volume penalty of only $10 \%$.
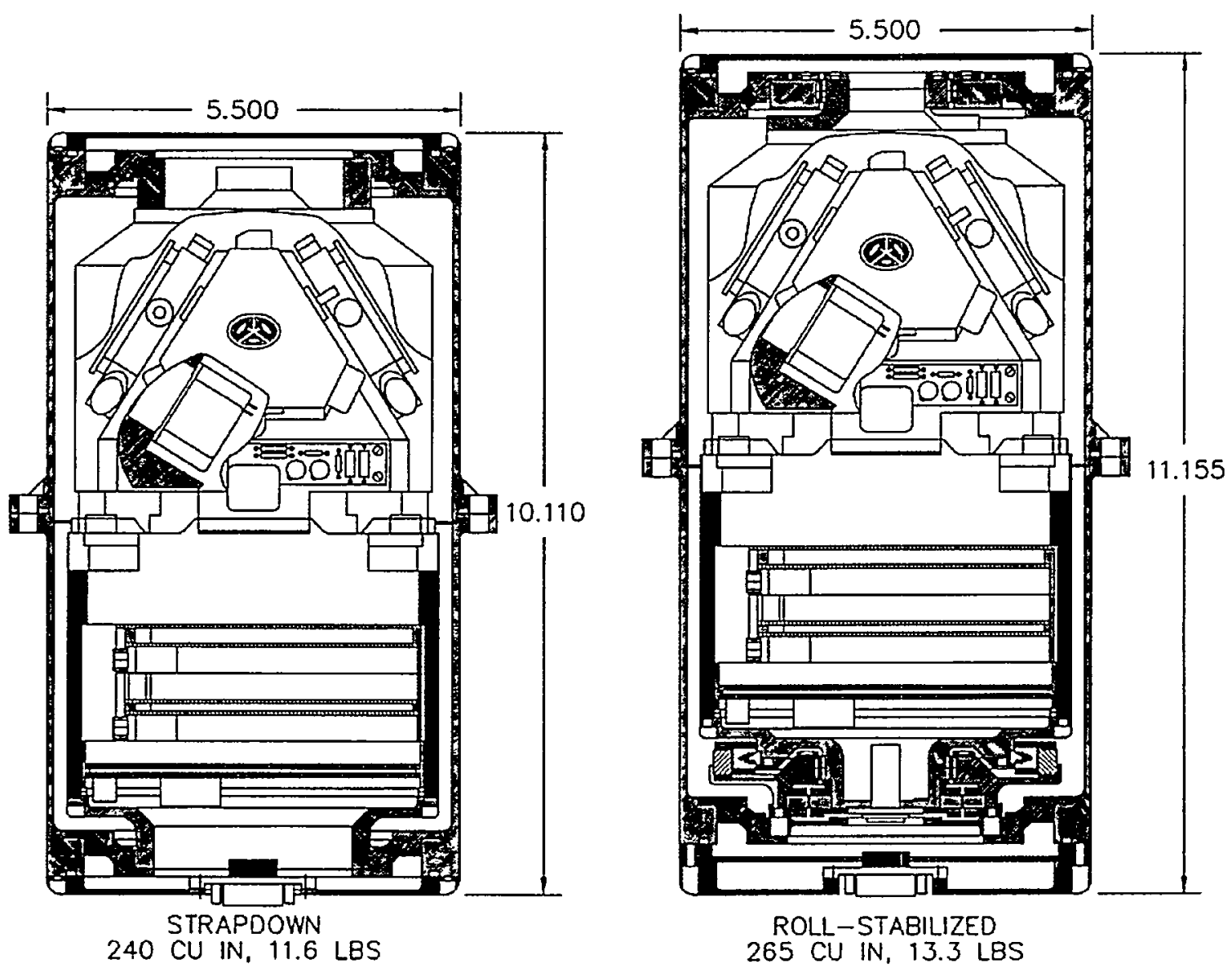

Figure 3: Strapdown RLGA and Roll-isolated RLGA-RIMS 
GLN-200: The GLN-200, a roll-isolated Litton LN-200 IMU, is a recent example illustrating more significant size and weight penalties. The GLN-200 has about three times the volume ( $100 \mathrm{cu}$ in vs. $35 \mathrm{cu}$ in) and weight $(5.6 \mathrm{lb}$ vs. $1.5 \mathrm{lb}$ ) of the strapdown LN-200 (Figure 4 below). In general, the more compact the strapdown IMU, the higher the size and weight penalty for roll-isolation.

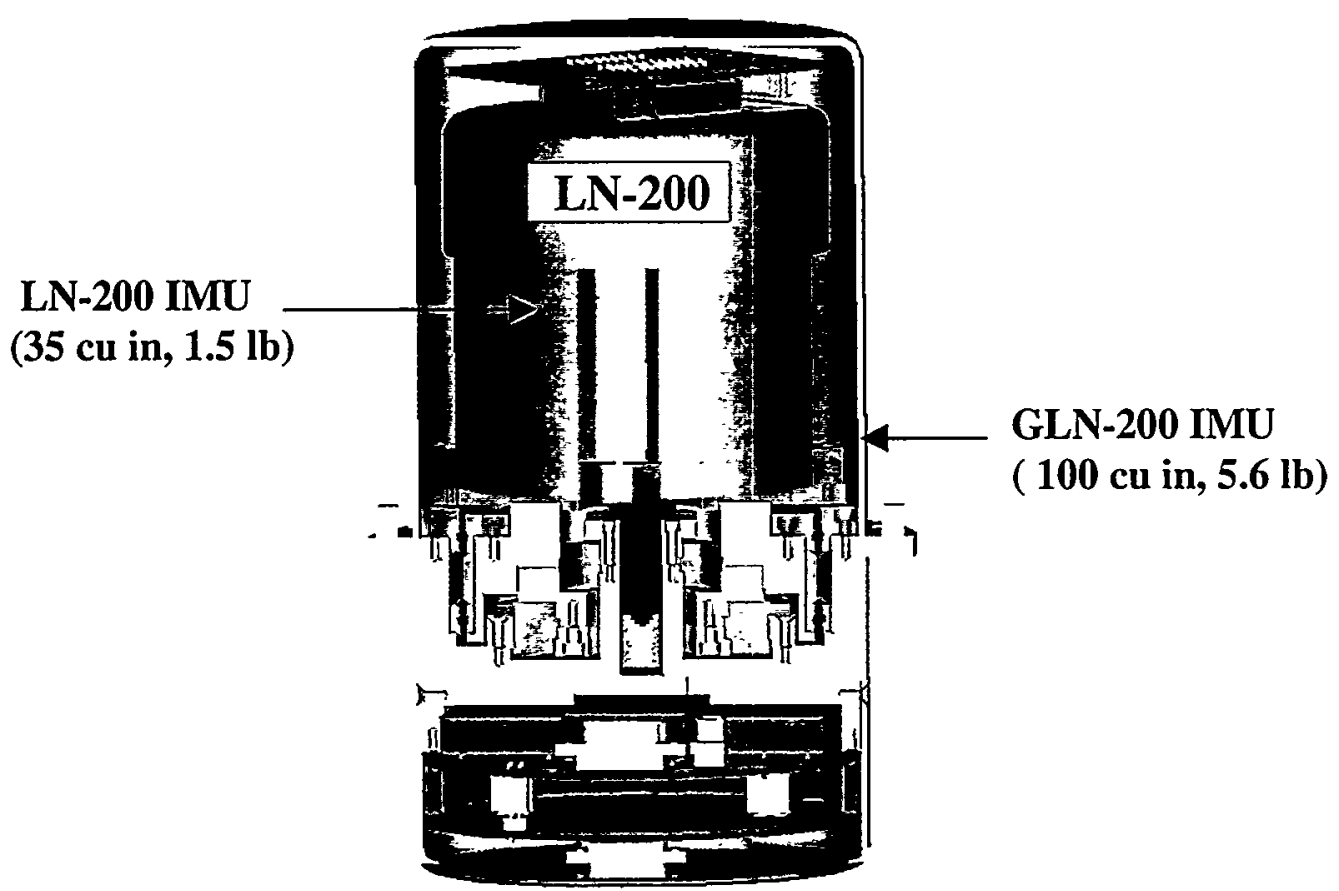

Figure 4: GLN-200 IMU Cutaway

Increased Power Requirement - The increased power required by gimbal-specific circuitry (i.e. motor drives, control processor, resolver excitation, R/D conversion, A/D conversion, and gimbal control logic, resulted in a twofold increase in power dissipation (21 watts vs. 10 watts) for the GLN-200 over the LN-200 at low G levels. However, the additional gimbal torque motor power required in high- $G$, high spin rate environments can increase tenfold over that required at low Gs. Fortunately, these severe environments are generally of short duration.

Bearing Resonance - The high-Q resonance of the gimbal bearings represents both a noise source, and vibration input multiplier, which can degrade performance and potentially damage inertial instruments if not attenuated. The use of vibration isolators has successfully mitigated these problems but with a weight and volume penalty.

Cost - Prior to the development of Litton's LN200 IMU, the inertial instruments and strapdown IMUs incorporated into roll-isolated systems were produced in small quantities with attendant high costs. For example, the cost of a strapdown RLGA, in quantities of ten, was over $\$ 200,000$. As a result, the incremental cost of the gimbal components required to roll-isolate the IMU amounted to much less than a one-percent penalty. Also, so few roll-isolated IMUs of any particular design were ever fabricated that development costs of several hundred thousand 
dollars for each design were dominant and were never amortized. Because of volume production, the LN200 is available for $\$ 10,000$. Estimates of incremental production costs of adding the gimbal components, isolators, additional electronics, and assembly are $\$ 3000$ per system in volume production.

\section{Study of Possible Influence of IMU Roll-isolation on Vehicle Dynamics}

To first order, the roll-isolation feature has no impact on vehicle dynamics. Inertial stabilization of the gimbal means that average net torque on the gimbal, as well as equal-and-opposite reaction torque on the vehicle, are essentially zero. However, especially sensitive applications exist, in which even second-order effects might be a concern. To address this issue, a computer simulation of a roll-isolated IMU (a GLN-200) in a spinning, coning, exoatmospheric RV was developed by Beverly R. Sturgis of Sandia's Aerosciences and Computational Fluid Mechanics Department. Beverly used the simulation to investigate two categories of IMU-related effects including:

Normal Operation:

- Change in effective vehicle moments-of-inertia due to IMU roll-isolation.

- Momentary (less than $1 \mathrm{sec}$.) non-zero reaction torques due to finite bandwidth of the gimbal servo.

Abnormal Operation:

- Worst case malfunction scenario: A short or other electrical failure causes the gimbal to spin up uncontrollably to its maximum possible angular velocity relative to the vehicle.

The simulation was developed using Autolev ${ }^{\mathrm{TM}}$ symbol manipulation software (Online Dynamics, Inc.). Only the exoatmosperic portion of flight was modeled, as the IMU effects on vehicle dynamics are known to be small, and deemed more likely to be observed during and/or following the extended period of torque-free motion. Two RVs were simulated, and results compared. RV-A was a "standard" RV, with typical mass properties. RV-B was identical, but contained a roll-isolated GLN-200 IMU. The misalignment of the GLN-200 gimbal axis relative to the spin axis of the RV as well as the location of the GLN-200 CG (center-of-gravity) relative to that of the RV were set up as variables, so their importance could be investigated during the course of the study. Internal forces and torques between RV-B and the IMU were modeled, and included gimbal bearing friction, gimbal motor torque, and constraint forces and torques at the IMU mount. Although the roll-isolation feature assures that the net torque on the IMU gimbal as well as the corresponding reaction torques on the vehicle average to zero, momentary (fractional second duration) imbalances may exist due to the finite frequency response of the gimbal stabilization servo. The gimbal servo was modeled as a second-order control system with a bandwidth of $10 \mathrm{~Hz}$ and a damping factor of 0.7 . Gimbal bearing friction torque was modeled as 
a sinusoidally varying (between 8 and 12 in-oz) function of the relative angle between the stabilized IMU gimbal and the spinning RV. This variation is typical, and arises from slight manufacturing variations in the gimbal bearing supports. The two categories of results are discussed below.

Normal Operation: At the start of the simulation, both RVs were spun up (by identical torques) to approximately 2 rps over a period of 5 seconds. Sufficient cross-axis angular velocity was imparted to produce a cone angle of 2 degrees. The simulation time of flight was 1800 seconds. Figures 5 and 6 below contain plots of roll and coning motions for both RVs, and are typical of the simulation results.

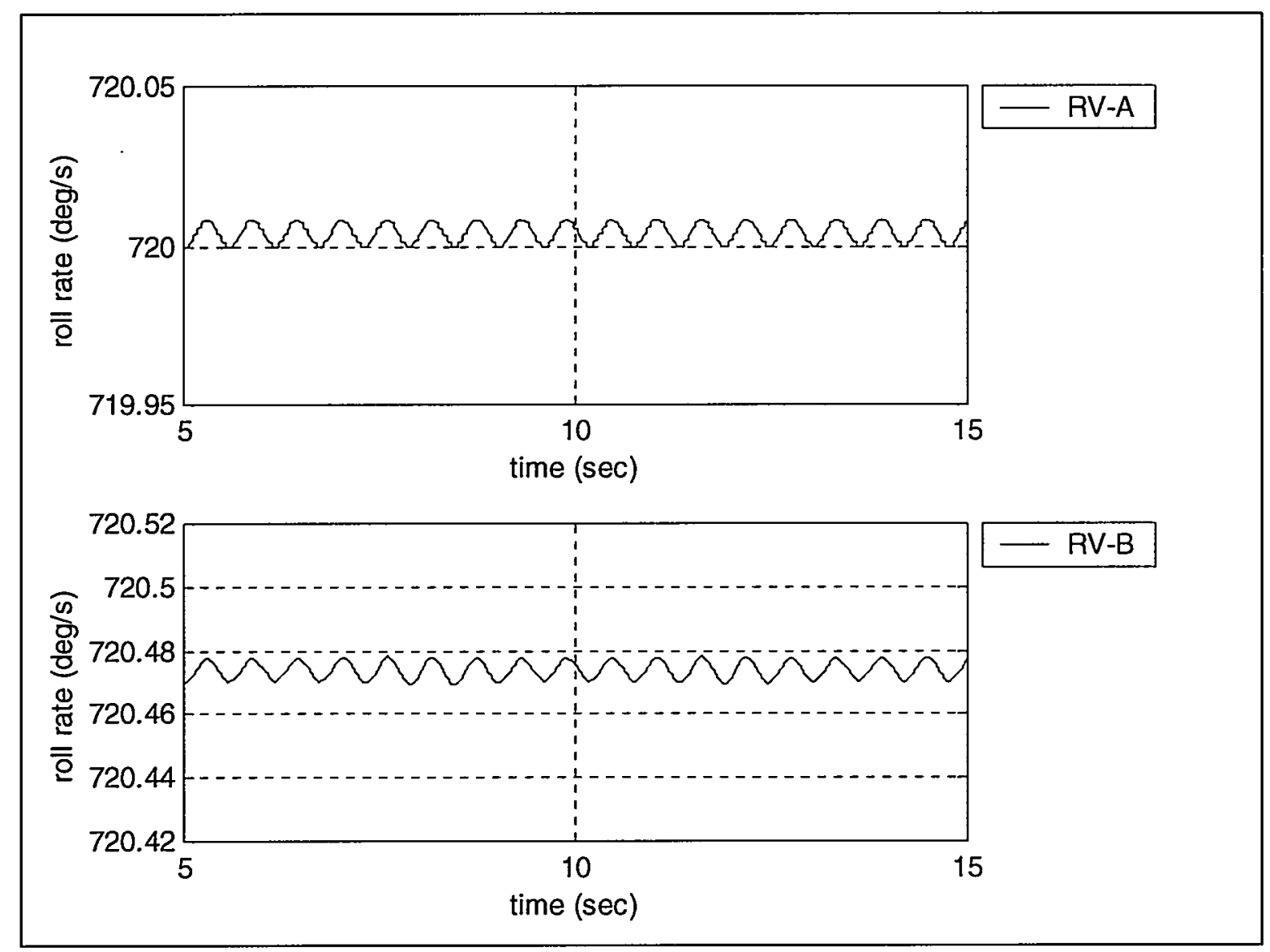

Figure 5 : Simulation Results - RV Spin Rates, Exoatmospheric Flight

The plots of Figure 5 show that at spinup, RV-B spun up to a slightly faster average angular velocity, by about 0.47 degree/sec, than that of RV-A. This is because the gimbal of the GLN-200 in RV-B is roll-stabilized (does not spin up) and does not contribute to the roll moment of inertia of RV-B. Thus the angular inertia of RV-B about its spin axis is slightly less than that of RV-A and its response to the spinup torque is greater. This effect of IMU roll-isolation on spin rate (less than $0.1 \%$ ) is very much smaller than the accepted spin up variability for a typical 
$\mathrm{RV}$ deployment system. The ripple on the roll rate is due to slight vehicle asymmetries such as CG offset.

Relative to the effect on vehicle roll inertia, other IMU-related effects (due to misalignments, finite servo bandwidth, etc.) were negligible.

Similarly, Figure 6 illustrates the effect of IMU roll-isolation on coning conditions.

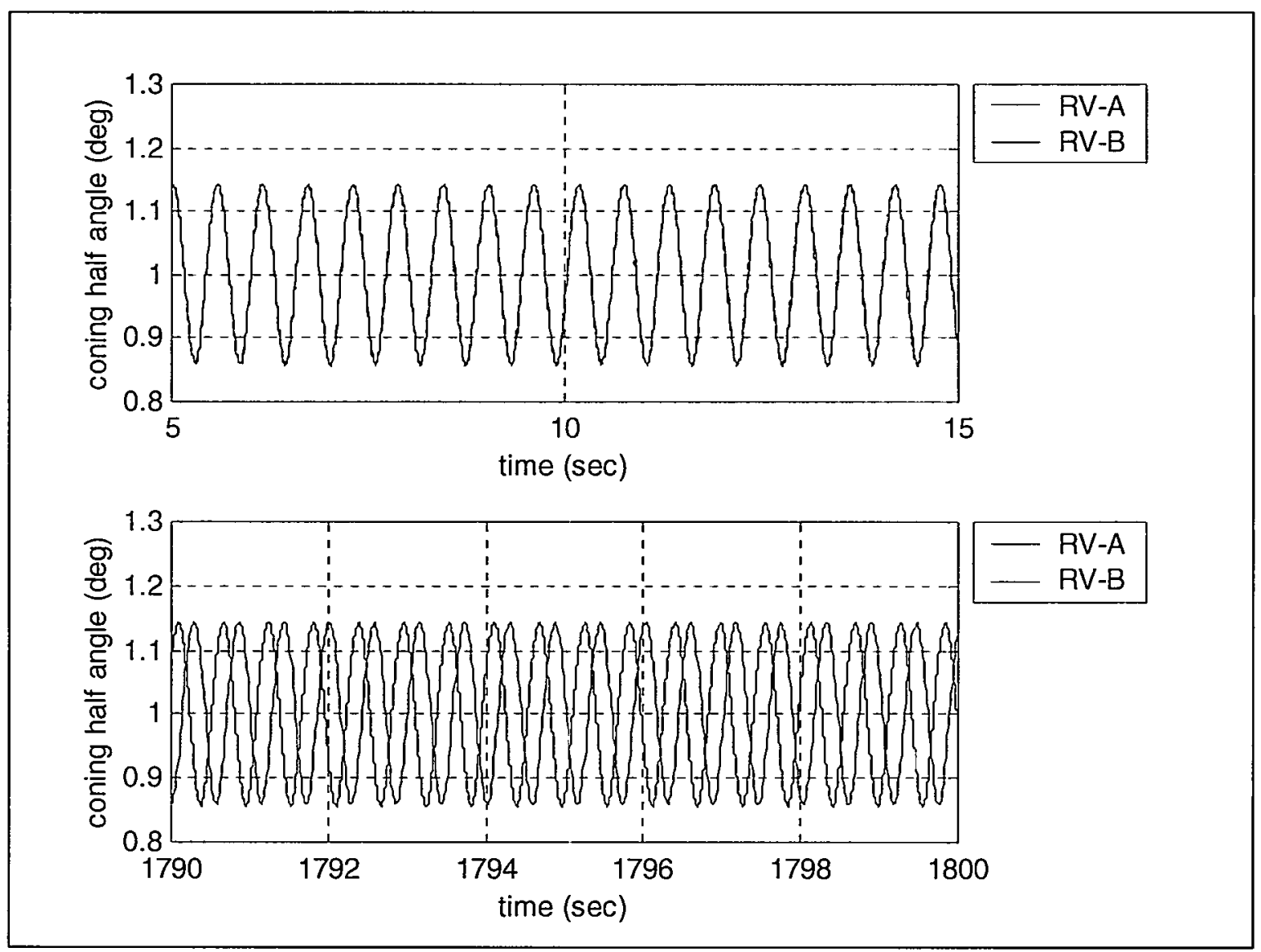

\section{Figure 6 : Simulation Results - Normal Operation: RV Cone Angles near Start and End of Exoatmosperic Flight}

It was assumed that RV-A and RV-B are deployed with the same cross-axis angular velocity. Therefore, the coning amplitudes in Figure 6 are the same, as expected, given the fact that the angular momenta about corresponding axes are the same. Again, the ripple on the coning is due to normal vehicle asymmetries. During the flight, a phase difference between the RV-A ripple and RV-B ripple develops at a rate equal to the difference in spin rate $(0.47$ degrees $/ \mathrm{sec})$. 
In summary, the predominate effect of IMU roll-isolation on RV dynamics is a slight increase in spin rate which is well within typical bounds of acceptable variation.

Abnormal Operation: A worst-case electrical failure, such as loss of gyro feedback to the gimbal servo, which places the full battery voltage across the gimbal motor, is assumed. Although such a failure has never ocurred in any of the twenty-seven flight tests involving Sandia roll-isolated IMUs, it has been known to occur in other gimballed, inertially stabilized systems. In this failure mode, the gimbal of the GLN-200, instead of being inertially stabilized, would "run away", spinning up to about 20 rps. This maximum angular rate, determined by laboratory experiment, is limited by bearing friction, viscous drag, motor back-EMF (electromotive force), and available battery voltage. As the IMU gimbal spins up, reaction torque on the vehicle would cause it to spin up or down depending on the direction of the gimbal runaway. In either case, the change in vehicle spin rate would be around five degrees per second or about $0.7 \%$. This was calculated from conservation-of-momentum considerations and verified by the simulation. A spin rate variation of this amount, while undesirable and much larger than the variation expected from normal IMU operation, is within typical RV spin rate tolerances. As in the normal case, the cone angle amplitude is unaffected, with the spin rate change showing up as an equivalent rate of change in phase difference between the cone angles of RV-A and RV-B.

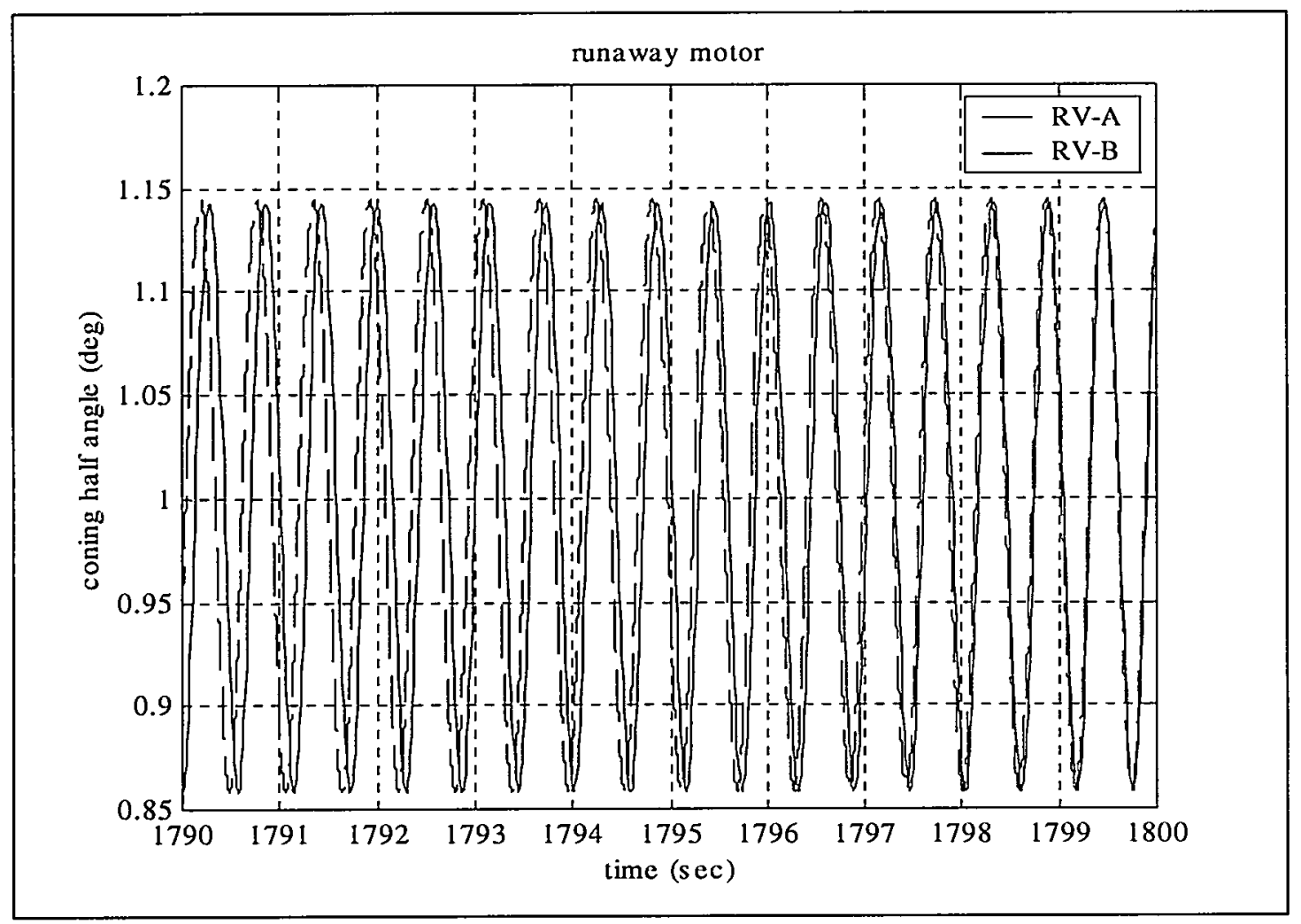

Figure 7 : Simulation Results - IMU Spinup Failure: RV Cone Angles near End of Exoatmosperic Flight 
This page intentionally left blank 


\section{DEVELOPMENT TESTING}

Acceleration and Spin Rate Testing - In addition to typical vibration and temperature testing, roll-isolated IMUs are subjected to acceleration and spin rate testing. For re-entry vehicle instrumentation and maneuvering RV guidance applications, a concern is that under high-G reentry decelerations, loading of the roll-isolated assembly on its support bearings will increase the bearing friction above the torque capability of the motor, and roll isolation will be lost. The capability of the torque-motor to overcome bearing friction is also spin rate dependent because the back-EMF of the torque motor reduces the maximum current which can be supplied from a fixed 28 VDC supply. To provide an estimate of the spin rate capability of a roll-isolated IMU, and its dependence on acceleration, the IMU is mounted on a high-g centrifuge with the roll-axis parallel to the centrifuge spin axis. The maximum spin rate of the gimbal assembly when the gimbal torque-motor is driven from a $28 \mathrm{VDC}$ supply is then recorded at increasing g-levels. Tests conducted on the GLN-200 indicated a 22 rps spin rate capability at low Gs decreasing to 9 rps at 150 Gs.

New Test Capability Under Development - When a roll-isolated IMU is operating in the stabilized mode and subjected to high Gs on a centrifuge, the test environment may not represent the expected flight environment. For example, when attempting to determine the IMUs lateral gcapability, the centrifuge spin rate at a particular G-level will not be equal to the expected vehicle spin rate during flight. To provide a more flexible and realistic test capability, a centrifuge-mountable spin assembly is being designed to allow independence of the spin rate and acceleration inputs. The assembly is designed to allow testing at spin rates up to $25 \mathrm{rps}$ at accelerations as high as 150 Gs. Figure 8 below is a cutaway drawing of the assembly.

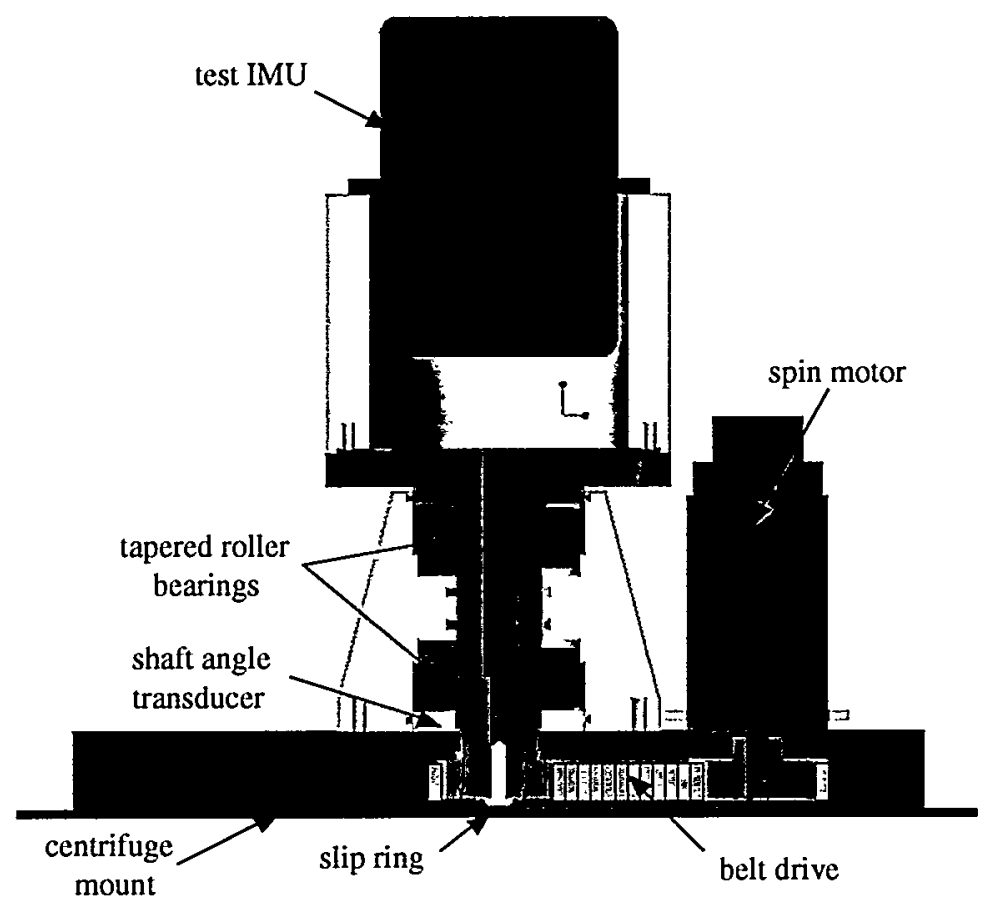

Figure 8 : Spin/Acceleration Test Assembly 
This page intentionally left blank 


\section{PRESENT IMU DEVELOPMENT EFFORTS}

QLN-2000 - A modification of the GLN-200 is in development for a re-entry vehicle (RV) instrumentation application that requires the measurement of very low level accelerations during $\mathrm{RV}$ release and spin-up (tip-off errors). The accelerations of interest during $\mathrm{RV}$ release are well below the noise floor of the $\mathrm{LN}-200$ accelerometers. The application also requires the measurement of re-entry decelerations exceeding $100 \mathrm{Gs}$, far beyond the $40 \mathrm{G}$ range of the present $\mathrm{LN}-200$ silicon accelerometers. To meet these difficult resolution and dynamic range requirements for acceleration measurement, a triad of Allied Signal QA-2000 accelerometers was added atop the LN-200 IMU within the GLN-200 to create the QLN-2000. A significant advantage of placing the Q-flex triad on a roll-stabilized assembly, as opposed to a "strapdown" triad, is that the minute accelerations during RV spin-up and release are uncontaminated by angular and centrifugal accelerations associated with RV spin. In addition, the measured accelerations are readily resolved into lateral, and along-path velocity errors to determine their effects on RV dispersion at the re-entry pierce point. To provide sufficient lateral load capability for the taller and heavier roll-isolated instrument cluster in the QLN-2000, an additional ball bearing was added between the LN-200 and the QA2000 triad. A mounting flange was added near the CG of the QLN-2000 assembly. The QLN-2000 is shown in Figure 9 below.

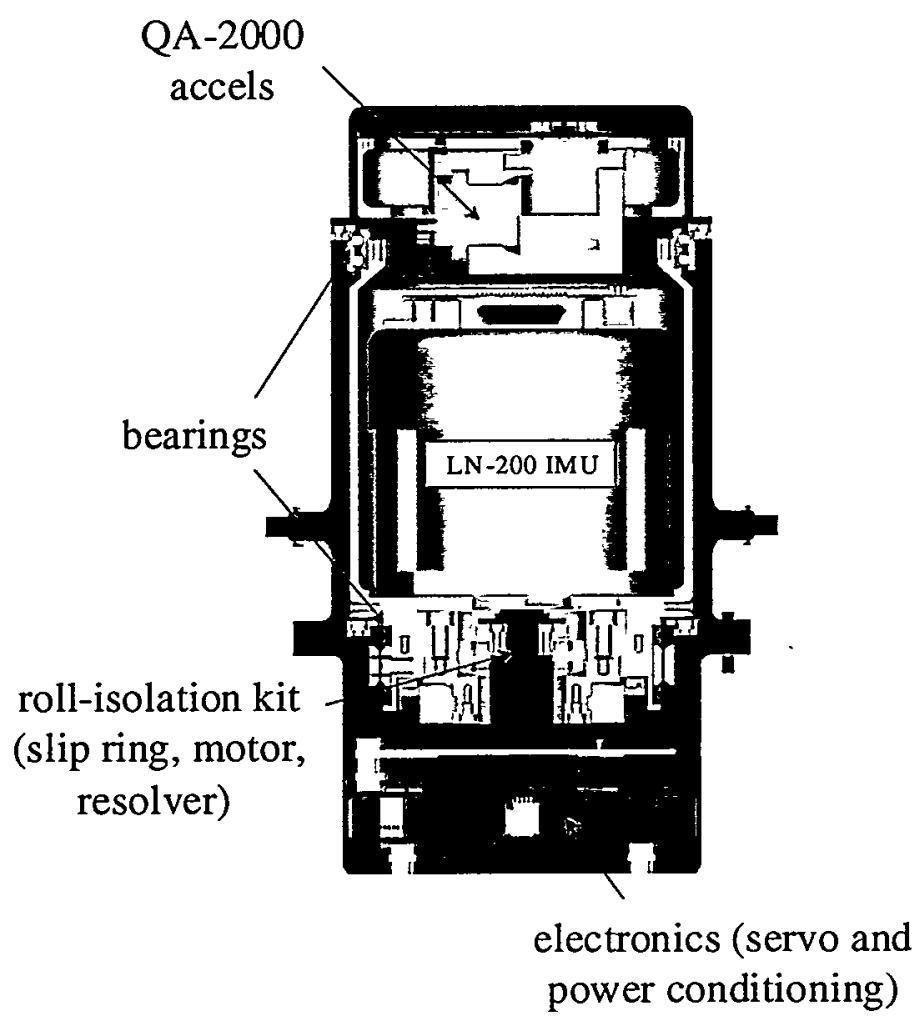

Figure 9 : QLN-2000 IMU Cutaway 
GLN-MAC and QLN-MAC - The Sandia Miniature $\underline{\text { Airborne }}$ Computer (SMAC), illustrated below in Figure 10, is being developed for a variety of size-sensitive applications. This singleboard (4.5" dia.) computer will be incorporated into the GLN-200 and QLN-2000 IMUs to provide a full inertial navigation system (INS) capability. These INSs will be designated GLNMAC and QLN-MAC, respectively. A major driver for developing the INS capability is the desire to reduce TM (telemetry) bandwidth requirements for reentry vehicle testing. The calculated position and attitude from an INS may be transmitted at a much lower rate than that required for raw IMU data of the same accuracy potential. Also, trajectory reconstruction accuracy is less sensitive to TM dropouts of INS data than it is to dropouts of IMU data. To ensure compatibility with existing inertial navigation software and development tools, the SMAC will have many features, including a MC68040 CPU, in common with the SANDAC V flight computer, which has been the workhorse for Sandia aerospace programs. The SMAC will have four serial ports for data communications, as well as discrete input/output for use in sending commands to rocket and reentry vehicle control systems.

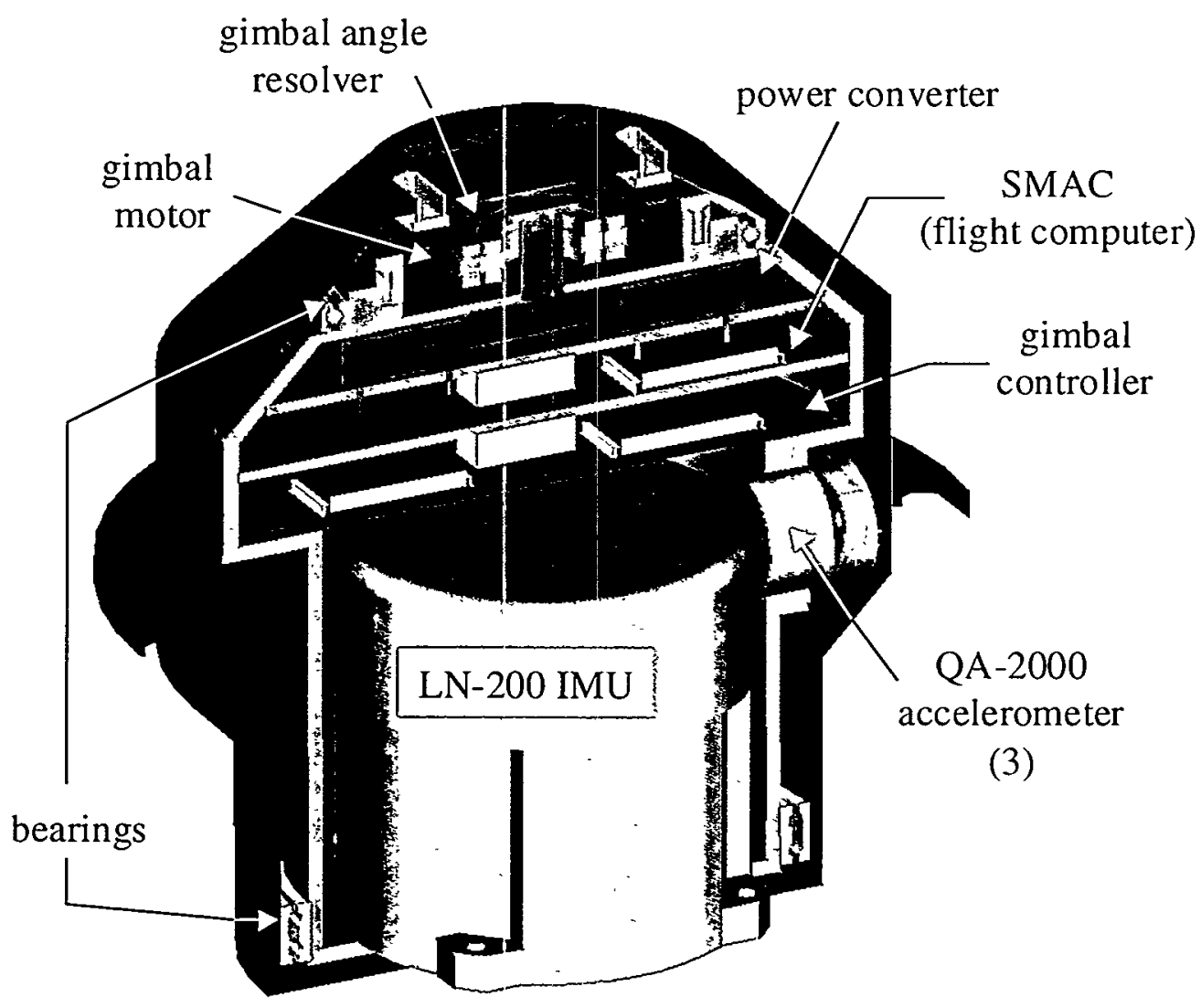

Figure 10 : QLN-MAC INS Cutaway 


\section{RECENT FLIGHT TEST RESULTS}

Energetic Kill Vehicle/Ground-Based Interceptor Program (EKV/GBI) - Sandia National Laboratories provides target vehicles for the EKV/GBI program in support of the National Missile Defense (NMD) program and the US Army Space and Missile Defense Command (USASMDC). Targets are launched from Vandenberg Air Force Base (VAFB) for the purpose of being intercepted by an EKV launched from the down-range Kwajalein Missile Range. The target vehicles are equipped with an attitude control system (ACS) designed to implement prescribed body dynamics. These spin-stabilized vehicles are equipped with GLN-200 IMUs that provide the inertial sensor data used by the ACS for control. The GLN-200 inertial sensor and resolver-to-digital (R/D) data is also used to generate relative attitude and absolute angular body rate information throughout the flight. Roll isolation of the inertial instrument cluster is an important requirement for this application. Prior to intercept, the GLN-200 IMU must operate for up to 25 minutes in the rapidly spinning vehicle. Without roll-isolation, the cumulative system errors could easily grow by an order of magnitude.

Three EKV/GBI Integrated Flight Test (IFT) target vehicles have flown with GLN-200 systems onboard: IFT-3, IFT-4, and IFT-5. These missions flew in October 1999, January 2000, and July 2000 , respectively. On each mission the GLN-200 worked as designed and achieved all preflight objectives. In addition to the navigation and ACS functions, the GLN-200 accelerometers were used to determine the relative separation velocity of the target vehicle with respect to the booster at the time of target vehicle deployment. Post flight reviews of the associated telemetry records indicate that the GLN-200 performed properly on all three vehicles.

W87 Joint Test Assembly (JTA) Instrumentation Development Flight-1 (IDF-1)- In support of nuclear stockpile surveillance requirements, the Department of Energy regularly conducts test flights of nuclear weapons systems. Typically these tests involve removing portions of the weapon and inserting specialized test instrumentation designed to record and telemeter information regarding the delivery vehicle dynamics and subsystem performance. The IDF-1 test vehicle flew in March, 2000. Instrumentation included a QLN-2000 IMU, SANDAC flight computer, and a Rockwell GPS receiver. Objectives for this system were to provide trajectory scoring information for the entire trajectory, and high-fidelity body dynamics measurements around the time of vehicle separation from the booster system. In the QLN-2000 IMU, both an LN-200 IMU and QA-2000 accelerometers are roll-isolated. In addition to the LN-200 inertial data, multi-range analog-to-frequency converters provided concurrent deltavelocity measurements for the QA-2000 accelerometers (for ranges of $+/-1.0 \mathrm{G},+/-30.0 \mathrm{G}$, and $+/-125.0 \mathrm{G}$ ). At the time of publication of this report, analysis of IMU flight data was just beginning. However, a quick look at roll-isolation performance was completed. The roll isolation system was required to function from the time of vehicle deployment and spin-up, through reentry and to water impact. Prior to reentry, the gimbal servomechanism maintained complete roll-isolation, requiring only $4.5 \mathrm{VDC}$ of gimbal motor drive voltage. During reentry, however, it was noted that as the spin rate climbed to over $10 \mathrm{rps}$ and the acceleration to over $50 \mathrm{Gs}$, the motor voltage required for roll-isolation reached the available supply voltage (28 VDC). During the last two seconds prior to impact, the instrument cluster was observed to be rotating at approximately $1 \mathrm{rps}$, indicating that the required voltage exceeded the available voltage by an estimated 1 volt. Although the QLN-2000 instrument cluster was not completely roll-isolated 
during this time, the angular rate was well within the angular rate capability of the $\mathrm{LN}-200$ roll gyro ( 1000 degrees/sec), so navigation was not compromised. Future flights of this system will use a 40 VDC supply to provide additional gimbal control authority and margin for even higher vehicle spin rates should they occur. 


\section{REFERENCES}

1. S.M. Kohler, J.W. Hole, C.S. Field, Development of a Roll-Stabilized Measurement System for Reentry Vehicle Applications, Sandia Internal Memorandum RS 2326/0008, (Albuquerque, NM: Sandia National Laboratories, June 1977).

2. A.C.Watts, R.D.Andreas, A Single Gimbal/Strapdown Inertial Navigation System for use on Spin Stabilized Flight Test Vehicles, IEEE /ch1597-4/80/0000-0250 (Albuquerque, NM: Sandia National Laboratories, 1980).

3. R.D.Andreas, G.M. Heck, S.M. Kohler, R. Wardlaw, Jr., A Wellbore Inertial Navigation System for Directional Surveying, IEEE CH 1820-0/82/0000-0221 Position Location and Navigation Symposium, Dec. 1982. (Albuquerque, NM: Sandia National Laboratories, 1980).

4. J.J. Imbault, H. Tjulin, Incosym Inc., Westlake Village, CA; R. Kinney and S. Kohler, Sandia National Labs, Albuquerque, NM, Mini RIMS: A Miniature Roll Stabilized Inertial Measurement System, AIAA-86-2525-CP, Paper-AIAA Conference on Sounding Rockets, Balloons and Related Space Systems, October 28-30, 1986/Ocean City, Maryland.

5. RLGA - RLGA Final Technical Report, Honeywell Inc. Space Systems Group, January 1993.

6. DMARS - DMARS-A Users Manual, Rev A.1, (Albuquerque, NM: Sandia National Laboratories, October 1993).

7. M.E.Beader, J.E. Lucero, J.L. Wilcoxen, The GLN-200 (Gimbaled LN-200) A Roll-Isolated Inertial Measurement Unit for Spinning Vehicle Guidance, Navigation, and Control, SAND2000-0928 Export Controlled Information Printed April 2000. (Albuquerque, NM: Sandia National Laboratories, 2000).

8. Product Description of the LN-200 Family, Litton Systems, Inc., Guidance \& Control Systems Division, 5500 Canoga Avenue, Woodland Hills, California 91367-6698, September 1996 
This page intentionally left blank 


\section{DISTRIBUTION}

1 MS 0501 J. Rick Fellerhoff, 2309

3 MS 0501 A. Butch Cox, 2334

12 MS 0501 Mark E. Beader, 2334

1 MS 0501 Ragon D. Kinney, 2334

1 MS 0501 Joseph E. Lucero, 2334

1 MS 0501 Jeffrey L. Wilcoxen, 2334

1 MS 9013 John W. Liebenberg, 2266

6 MS 9013 Edward B. Talbot, 2266

1 MS 0986 Vincent P. Salazar, 2663

1 MS 9101 Curt H. Cofield, 8411

1 MS 0313 Robert E. Sheldahl, 15415

1 MS 0313 Robert M. Stearley, 15415

2 MS 0301 Earl E. Creel, 15426

1 MS 1174 Alfred C. Watts, 15426

1 MS 9018 Central Technical Files, 8945-1

2 MS 0899 Technical Library, 9616

1 MS 0612 Review and Approval Desk, 9612 For DOE/OSTI 